\title{
Presupplementary Motor Area Activation during Sequence Learning Reflects Visuo-Motor Association
}

\author{
Katsuyuki Sakai, ${ }^{1,2}$ Okihide Hikosaka, ${ }^{1}$ Satoru Miyauchi, ${ }^{3}$ Yuka Sasaki, ${ }^{3}$ Norio Fujimaki, ${ }^{3}$ and Benno Pütz ${ }^{4}$ \\ ${ }^{1}$ Department of Physiology, Juntendo University School of Medicine, Tokyo 113, Japan, 2Department of Neurology, \\ Division of Neuroscience, Graduate School of Medicine, University of Tokyo, Tokyo 113, Japan, ${ }^{3}$ Communications \\ Research Laboratory, Kobe 651-24, Japan, and 'Exploratory Research for Advanced Technology, Japan Science and \\ Technology Corporation, Kyoto 619-02, Japan
}

In preceding studies (Hikosaka et al., 1996; Sakai et al., 1998) we have shown that the presupplementary motor area (preSMA), an anterior part of the medial premotor cortex, is active during visuo-motor sequence learning. However, the paradigm required the subjects first to acquire correct visuo-motor association and then to acquire correct sequence, and it was still unknown which of the two processes the pre-SMA is involved in. To further characterize the role of pre-SMA, we have conducted another series of functional magnetic resonance imaging experiments using three learning paradigms. The three were the same in that they involved a visuo-motor association component, but they differed in terms of the involvement of sequential components; one involved no sequence learning, whereas the other two involved learning of motor sequence or perceptual sequence. Comparison of the learning conditions with the any-order button press condition revealed pre-SMA activation in all three paradigms. The pre-SMA activation remained unchanged during learning of visuo-motor associations but decreased during learning of sequences, suggesting that the pre-SMA is related to visuo-motor association rather than sequence. The decrease of pre-SMA activation in the sequential paradigms may reflect the process by which individual visuomotor associations were replaced by the formation of sequential procedural memory, which occurs outside the pre-SMA. Thus activation of the pre-SMA was related to the extent to which the task performance depended on conscious visuomotor associations.

Key words: presupplementary motor area; visuo-motor association; sequence; learning; functional magnetic resonance imaging; premotor cortex
The presupplementary motor area (pre-SMA) is an area located in the anterior part of the medial premotor cortex originally demonstrated in the monkey (for review, see Tanji, 1996). Subsequent functional imaging studies revealed the human homolog of the pre-SMA, which is located anterior to the coronal plane passing the anterior commissure (VCA), and have shown its involvement in higher-order aspects of motor control (Picard and Strick, 1996). Consistent with this idea, we found, both in monkeys and humans, that the pre-SMA was active in visuo-motor sequence-learning tasks (Hikosaka et al., 1996; Nakamura et al., 1998; Sakai et al., 1998). However, because the learning paradigm involved both visuo-motor association and sequence, it remained unresolved which components the pre-SMA is associated with. In addition, the paradigm comprised two sequential components: motor sequence and perceptual sequence. To determine whether the pre-SMA activation is related to visuo-motor association or sequence (motor or perceptual), we used three learning paradigms. These paradigms involved the same visuo-motor association components but differed in their sequential components: motor sequence, perceptual sequence without motor sequence, and no sequence.

\section{MATERIALS AND METHODS}

Subjects. Six normal human subjects participated in the study (five males and one female, ages 29-50 years, all right-handed). Informed consents

\footnotetext{
Received Oct. 7, 1998; revised Jan. 7, 1999; accepted Jan. 12, 1999.

Correspondence should be addressed to: Okihide Hikosaka, Department of Physiology, Juntendo University, School of Medicine, 2-1-1 Hongo, Bunkyo-ku, Tokyo 113, Japan.

Copyright (C) 1999 Society for Neuroscience $\quad 0270-6474 / 99 / 0001-\bullet \$ 05.00 / 0$
}

were obtained from all the subjects before the study. The experimental protocol was approved by the ethics committee of our institute.

Task procedures. The subjects, lying supine in the magnetic resonance imaging (MRI) scanner, saw four white rectangles arranged in a $2 \times 2$ matrix in which two circles in different colors (four possible colors) appeared simultaneously in different positions (four possible positions) (colors and positions together called "set"; Fig. 1a,b). They had to press the buttons on a plate that corresponded to the circles using the index and middle fingers of both hands (Fig. 1b).

During the test conditions, the subjects had to find the predetermined correct order of pressing the two buttons for a consecutive number of sets by trial and error (called "hyperset," 6 or 10 sets; for details, see Sakai et al., 1998). The rate of button presses was paced by tone stimuli at 1 set/sec. Three learning paradigms were used; position sequence task (Pos-Seq), color sequence task (Col-Seq), and color mapping task (ColMap) (Fig. 2a).

Pos-Seq is the same as used in the previous studies (Hikosaka et al., 1996; Sakai et al., 1998). The number of sets in a hyperset was 10 , and the 10 sets were presented in a fixed order. The position of the two circles within $2 \times 2$ matrices for each set was the same across trials, whereas

This article is published in The Journal of Neuroscience, Rapid Communications Section, which publishes brief, peerreviewed papers online, not in print. Rapid Communications are posted online approximately one month earlier than they would appear if printed. They are listed in the Table of Contents of the next open issue of JNeurosci. Cite this article as: JNeurosci, 1999, 19:RC1 (1-6). The publication date is the date of posting online at www.jneurosci.org.

http://www.jneurosci.org/cgi/content/full/2875 

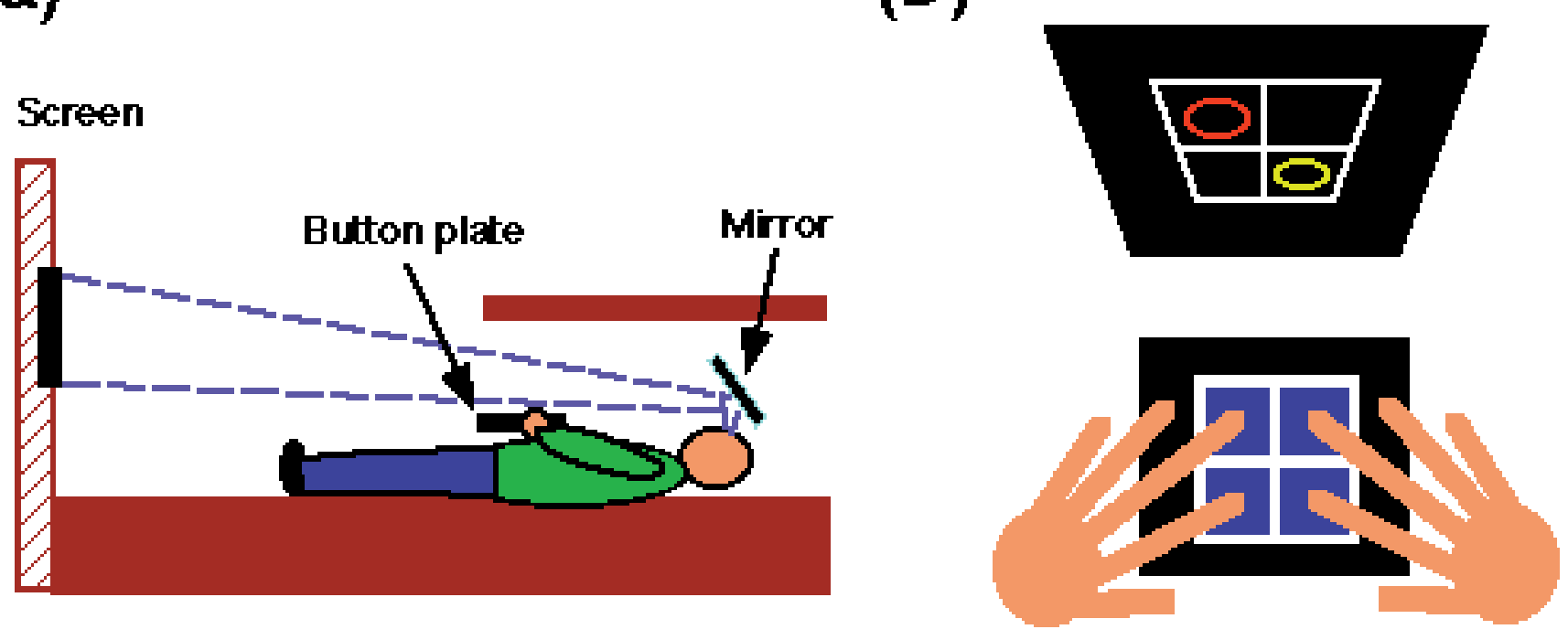

Figure 1. $a$, The subjects lay supine in the MRI scanner and saw the screen through the mirror. They held a button plate on which four button switches were attached. $b$, On the screen were presented $2 \times 2$ matrices in which two color circles appeared. The subjects had to press the two of the four buttons corresponding to the circles, using the same finger for each one of the four positions.

their color was changed from trial to trial. The correct order of button presses was determined by the position of the circles in each set, and the subjects were asked to use the same finger for each button corresponding to one of the four positions of the circles (Fig. 1b). Therefore, the correct order of finger movements was fixed in Pos-Seq for every trial of a hyperset. In other words, the subjects learned both the motor and perceptual (position) sequences.

In Col-Seq, 10 sets were presented also in a fixed order in a hyperset. However, the correct order of button presses was determined by the color of the circles for each set, which was the same across trials. In contrast, the position of the circles was changed from trial to trial. Therefore, the correct order of finger movements was also changed for every trial of a hyperset. Thus, the subjects learned the perceptual (color) sequence but not the motor sequence.

In Col-Map, the correct order of button presses was also determined by the color of the circles. However, unlike in Col-Seq, the number of sets in a hyperset was six, and the six sets, having different combinations of colors, were presented in a random order for each trial. The correct order of button presses was determined uniquely for each combination of colors. Therefore, no sequence learning was involved in Col-Map, but each combination of colors had to be correctly mapped to the order of button presses.

In short, the three paradigms were the same in that the subjects had to find the correct order of button presses for each set (learning of visuomotor association), whereas they differed such that Pos-Seq involved both the motor and perceptual sequence, Col-Seq involved only the perceptual sequence, and Col-Map involved no sequence (Fig. $2 b$ ).

During the control conditions, the subject pressed the buttons for the two circles in any order, and the computer determined randomly whether the button press was correct. The error rate was programmed to match that of the test conditions. Thus the subjects experienced the same sensorimotor processes as in the test conditions, but no learning was involved (called "pseudo-learning"; see Sakai et al., 1998). Thus, comparison of the neural activities between the two conditions would selectively reveal learning-related activation.

Functional MRI experiments. We used a 1.5 tesla whole-body scanner (Siemens Vision, Erlagen, Germany) equipped with a circular-polarized head coil. First, T1-weighted sagittal anatomical brain images [FLASH; repetition time (TR), echo time (TE), and inversion time (TI), 2800, 4, and $300 \mathrm{msec}$, respectively; flip angle (FA), $15^{\circ}$; matrix, $256 \times 256$, field of view (FOV), $256 \times 256 \mathrm{~mm}$; and slice thickness, $1 \mathrm{~mm}$ ] were obtained for each subject to determine the anatomical landmarks. Subsequently, three functional MRI (fMRI) experiments were conducted, each using one of the three learning paradigms as a test task and pseudo-learning as a control task. The order of the three experiments was counterbalanced across the six subjects. Each experiment consisted of eight alternating blocks of the test and control conditions, each one of them lasting $42 \mathrm{sec}$, and, between the two conditions, instruction was presented on the screen for $6 \mathrm{sec}$, indicating the next condition. Each subject continued to learn the same hyperset throughout the eight test blocks. In the meantime, a time series of 128 scans separated by $6 \mathrm{sec}$ was performed for each experiment. In each scan, a set of 10 axial $\mathrm{T} 2 *$-weighted gradient-echo echo-planar images (TR, TE, and TI, 6000, 66, and $300 \mathrm{msec}$, respectively; FA, $90^{\circ}$; FOV, $220 \times 220 \mathrm{~mm}$; matrix, $128 \times 128$; and slice thickness, $5 \mathrm{~mm}$ ) was collected parallel to the anterior commissureposterior commissure line.

Data analysis. After motion correction (AIR 3.0; Woods et al., 1992) and spatial smoothing with a Gaussian filter $(4.5 \mathrm{~mm}$ full width, halfmaximum), the time series of signal intensity (SI) data for each experiment was cross-correlated with an idealized boxcar reference function shifted for one data point to account for the hemodynamic delay. If the correlation coefficient calculated for a pixel was $>0.35$, the pixel was determined to be significantly more active (corresponding to $p<0.0001$, uncorrected). Then, for each individual subject, we determined the region of interest (ROI; Fig. 3a, region encircled by the green lines) for the pre-SMA to include all the pixels active in either of the three experiments within the medial premotor cortex above the cingulate sulcus and anterior to the VCA line (Fig. 3a, yellow lines). Thus we compared the activity of the identical brain areas for the three experiments.

The relative SI increase for each of the eight test blocks from the preceding and following control blocks $(\Delta \mathrm{SI})$ was calculated for this ROI of the pre-SMA (for details, see Sakai et al., 1998). To account for the difference in the speed of learning among the subjects and also among the tasks, we classified each of the eight test blocks into one of the four learning levels based on the rate of correct performance. The correct performance rate was calculated by dividing the number of correctly performed sets by the total number of performed sets (usually 42) and was expressed as a percentage. Blocks with a correct performance rate $<70 \%$ were categorized into level 1 , those with $70-80 \%$ into level 2 , those with $80-90 \%$ into level 3, and those $>90 \%$ into level 4 . Subsequently, the mean $\Delta$ SI was calculated for each learning level for each subject. Thus the time course of the pre-SMA activation can be shown as the mean $\Delta$ SI expressed as a function of the learning level.

To test the difference in the time course of the pre-SMA activation across the three experiments, repeated measurements of ANOVA were performed on the $\Delta$ SI using two within-subjects factors: learning level (Level) and task paradigm (Task). 
(a)

\section{Visual stimuli}

\section{Pos-Seq}

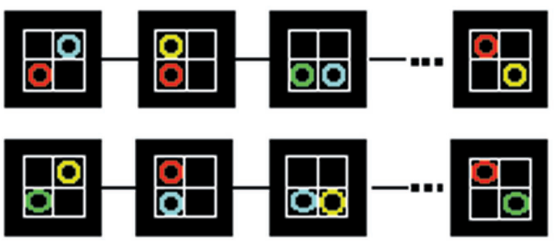

\section{Col-Seq}

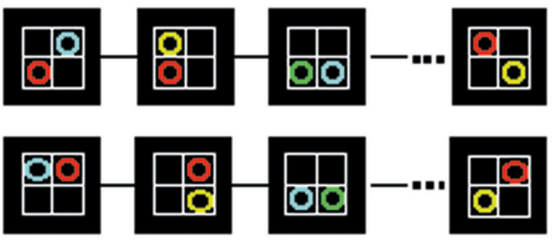

\section{Col-Map}

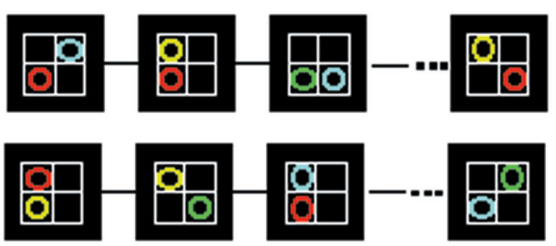

\section{Correct response}

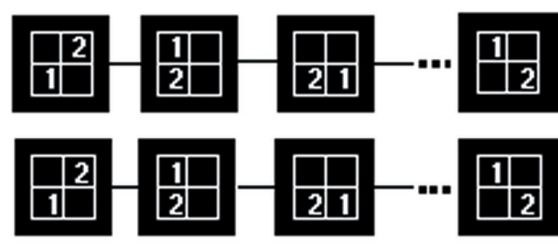

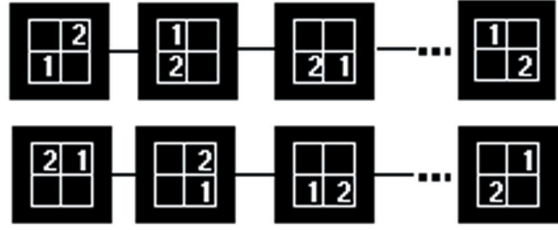

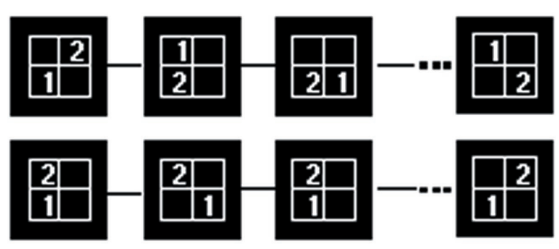

Figure 2. $a$, Examples of two successive trials for position sequence (Pos$\mathrm{Seq}$ ), color sequence (Col-Seq), and color mapping tasks (Col-Map). The subjects had to find the correct order of button presses (shown on the right), which was predetermined by the position (Pos-Seq) or color (Col-Seq and Col-Map) of the circles. In Pos-Seq, for example, the correct order of button presses was bottom left $\rightarrow$ top right for the first set and top left $\rightarrow$ bottom left for the second set. In Col-Seq, the correct order was red $\rightarrow$ blue for the first set and yellow $\rightarrow$ red for the second set. In ColMap, the six sets (i.e., 6 possible color combinations) were presented in random order. For example, the correct order of button presses for the pair of red and blue circles was red $\rightarrow$ blue, but this pair appeared as the first set in the top trial, whereas it appeared as the third set in the bottom trial. Also note that the patterns of finger movements were fixed in Pos-Seq but were varied in Col-Seq and Col-Map. $b$, The three tasks were the same in that they involved learning of visuo-motor association (to find the correct order of button presses for each set), whereas they differed in terms of the involvement of sequential (perceptual and motor) components.

\section{RESULTS}

All the subjects showed improvement in performance from level 1 to level 4 during a single run of the experiment for all three learning paradigms. Although the number of sets in a hyperset was different among the three tasks (10 for Pos-Seq and Col-Seq, 6 for Col-Map), the learning performance as measured by the increase of the correct performance rate for the eight test blocks was not different (Fig. 3c). ANOVA applied to the correct performance rate of the six subjects revealed that both the main effect of Task and Task $\times$ Block interaction were not significant $\left(F_{(2,10)}=0.968 ; p>0.05 ;\right.$ and $F_{(14,70)}=1.81 ; p>0.05$, respectively).

For all three learning paradigms, significant activation was observed in the medial premotor cortex, lateral prefrontal cortex, and posterior parietal cortices (Sakai et al., 1998). The difference of activation pattern was observed in the location of the prefrontal cortex activity; its ventral part was more active in Col-Seq and Col-Map compared with Pos-Seq. An additional difference was found in the extent of activation for all the areas mentioned above; in general, wider areas were active in Col-Map compared with the other two.

The present study focused on the activation of the medial premotor cortex, which was nearly identical across the three experiments (Fig. $3 a$ ). The area was located above the cingulate sulcus and anterior to the VCA (Fig. 3a, yellow line), was thus identified as the pre-SMA. As shown in Figure $3 b$, the SIs for the pre-SMA were higher in the test conditions (gray areas) than in the control conditions, the difference of which $(\Delta \mathrm{SI})$ reflected the cognitive components required for learning. However the $\Delta$ SI decreased gradually across the eight blocks in Pos-Seq and Col- 


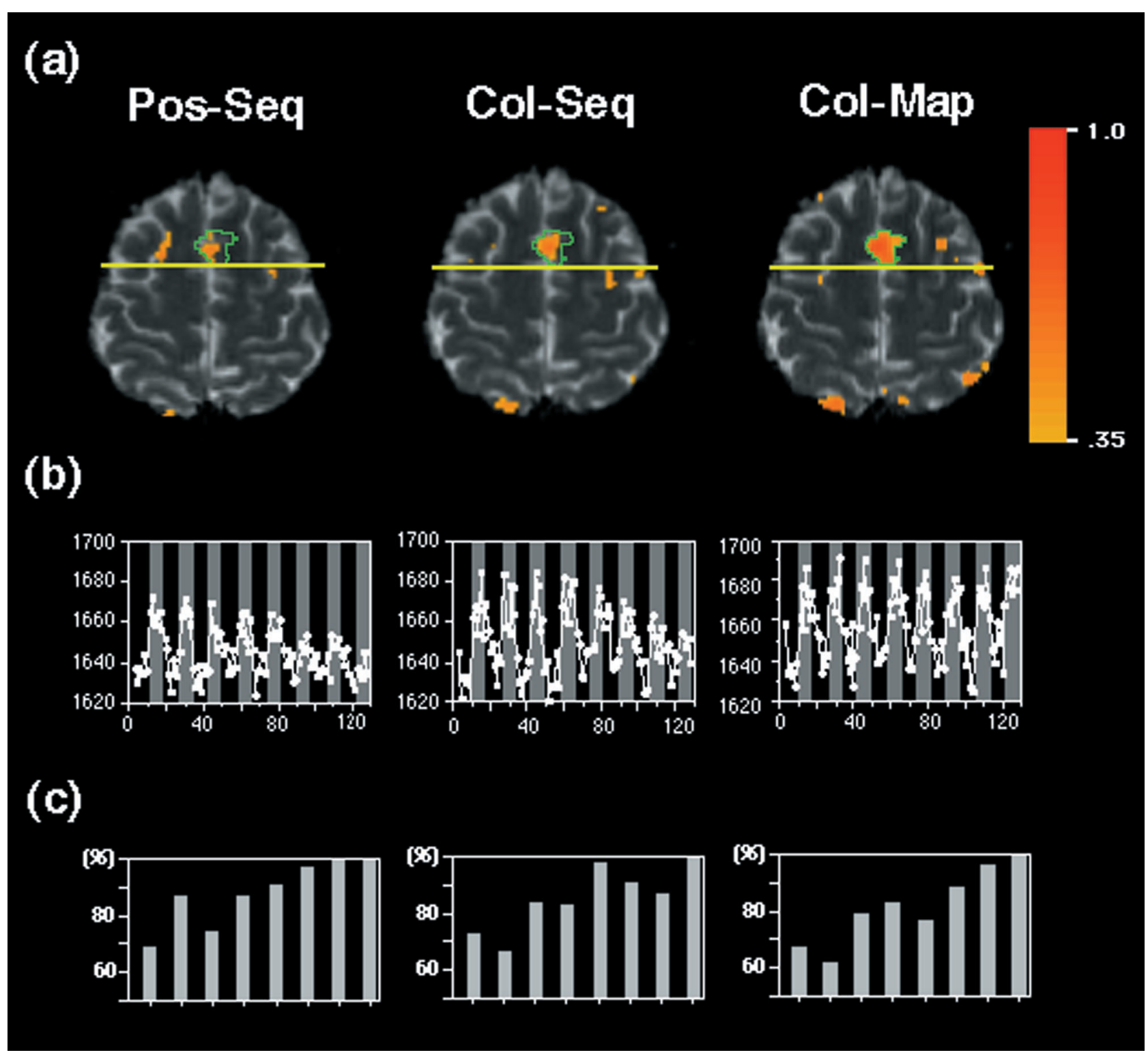

Figure 3. a, Activation maps from a representative single subject. The right hemisphere is shown on the left. The active pixels were determined as the ones with correlation coefficients $>0.35$ and were color-coded in orange-red according to the color bar on the right. In each subject, the ROI for the pre-SMA, encircled by the green line, was placed on the medial premotor cortex anterior to the VCA line (yellow line) to include all the pixels active in any of the three experiments. $b$, Time course of the mean signal intensity for the pre-SMA ROI during the three experiments. The eight blocks for the test condition are shown in gray. Note the difference between the three experiments. $c$, The performance of the subject was expressed by the correct performance rate (the number of correctly performed sets/the number of performed sets) plotted against the eight blocks.

Seq, whereas it remained unchanged in Col-Map, indicating the difference in the time course of pre-SMA activation. Because the improvement in performance was similar across the three tasks (Fig. 3c), the difference in the time course of pre-SMA activation would be attributable to the task requirements, not the difference in the learning performance.

To confirm this idea, the pre-SMA activation was calculated respectively for each one of the four learning levels (mean $\Delta \mathrm{SI}$ ). ANOVA applied to the mean $\Delta$ SI showed significant Task $\times$ Level interaction $\left(F_{(6,30)}=11.7 ; p<0.01\right)$, again indicating the difference in the time course of pre-SMA activation between the tasks. Post hoc testing (Tukey's honestly significant difference method) revealed that Level significantly affected $\Delta$ SI in Pos-Seq and Col-Seq $(p<0.01)$ but did not in Col-Map $(p>0.05)$. As shown in Figure 4, the mean $\Delta$ SI in Pos-Seq and Col-Seq decreased along with the progress in the learning level, whereas that in Col-Map remained unchanged.

\section{DISCUSSION}

The present study used three learning paradigms to answer the question, is the pre-SMA related to visuo-motor association or sequence? As shown in Figure 2b, Col-Map required the acquisition of visuo-motor associations, whereas Pos-Seq and Col-Seq required the acquisition of a sequence as well. The pre-SMA activation was commonly observed in all three paradigms, suggesting that this area is related to visuo-motor association rather than motor or perceptual sequence. Interestingly, the activity of the pre-SMA decreased gradually in Pos-Seq and Col-Seq. This 


\section{Pos-Seq}

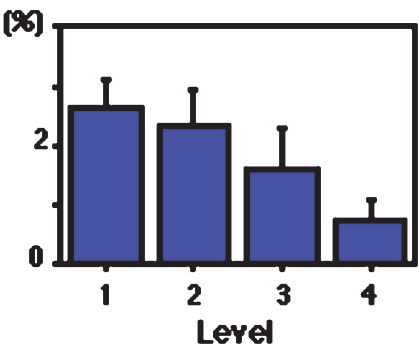

Col-Seq

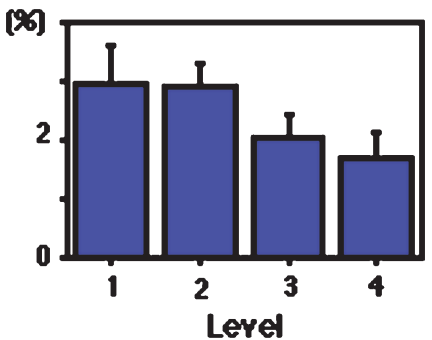

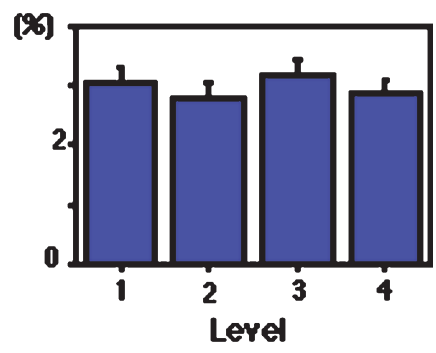

Figure 4. Time course of the relative SI increase $(\Delta \mathrm{SI})$ shown as a function of the learning level (mean of 6 subjects; error bar indicates SE). Level 1 indicates the early phase of learning, and Level 4 indicates the advanced phase. A decrease of $\Delta$ SI along with the progress in learning was observed in Pos-Seq and Col-Seq but not in Col-Map. may indicate that, in Pos-Seq and Col-Seq, the visuo-motor association processes operating on individual sets were taken over during learning by the sequence process operating on a whole hyperset. After acquisition of the sequential procedure, it was no longer necessary to consciously retrieve the visuo-motor association at each set, and therefore the pre-SMA activation was not required. By contrast, in Col-Map, the subjects had to retrieve the visuo-motor association at each set even at the late stage of learning, resulting in the persistent pre-SMA activation. This persistent pre-SMA activity despite the decrease in the number of errors would exclude the possibility that the activity merely reflected arousal or error correction.

The present results are consistent with the pre-SMA neuronal activities in monkeys performing a task similar to Pos-Seq (Nakamura et al., 1998): many pre-SMA neurons behaved in the same manner as in the present study, showing prominent discharge during new sequence learning, which decreased along with the progress in learning performance. Interestingly, at the initial phase of learning, most of the pre-SMA neurons discharged at every set (Nakamura et al., 1998, their Fig. 4), and the activity was usually observed between the stimulus presentation and the first button press during which the subjects tried to associate the visual stimuli with the correct motor responses. The fact that a single neuron discharged for any set would suggest that each pre-SMA neuron is not specialized for a particular type of visuo-motor transformation but is related, more generally, to the control of visuo-motor transformation (Rizzolatti et al., 1996). This idea might be supported by the anatomical finding (Luppino et al., 1993) showing that the pre-SMA receives inputs from the prefrontal and cingulate cortex and sends projections to the lateral premotor cortex, which is shown to be involved in visuomotor transformation (Wise et al., 1992).

Learning-related modulations of neuronal activity were also found in the supplementary eye field (SEF) of the monkey during a conditional oculomotor association task (Chen and Wise 1995). In the present tasks, although the subject had to press the two buttons in response to each visual stimulus, the second button to be pressed was automatically determined once the first button was selected. Therefore, the Col-Map can be thought to require essentially the same cognitive operations as the traditional conditional motor learning. Because we did not ask the subjects to fixate their eyes during learning, the activation of the medial premotor cortex observed in the present study might include the activity of the SEF in addition to that of the pre-SMA.

It is interesting that the pre-SMA activity decreased also in Col-Seq, in which the subjects had to change the pattern of movements for each trial. This suggests that perceptual sequence alone contributes to the procedural memory formation, as has been demonstrated by Fendrich et al. (1991).
As discussed, we consider that learning occurs in two stages, first acquisition of visuo-motor associations and then acquisition of the whole sequence, and the pre-SMA, which was active in the former stage, is related to visuo-motor association components. The sequential components may be subserved by the supplementary motor area proper (Tanji and Shima 1994) and basal ganglia (Kermadi and Joseph, 1995; Mushiake and Strick, 1995; Miyachi et al., 1997; Rauch et al., 1997) and possibly by the precuneus (Ghaem et al., 1997; Maguire et al., 1997; Sakai et al., 1998).

\section{REFERENCES}

Chen LL, Wise SP (1995) Neuronal activity in the supplementary eye field during acquisition of conditional oculomotor associations. J Neurophysiol 73:1101-1121.

Fendrich DW, Healy AF, Bourne LEJ (1991) Long-term repetition effects for motoric and perceptual procedures. J Exp Psychol Learn Mem Cogn 17:137-151.

Ghaem O, Mellet E, Crivello F, Tzourio N, Mazoyer B, Berthoz A, Denis M (1997) Mental navigation along memorized routes activates the hippocampus, precuneus and insula. NeuroReport 8:739-744.

Hikosaka O, Sakai K, Miyauchi S, Takino R, Sasaki Y, Pütz B (1996) Activation of human presupplementary motor area in learning of sequential procedures: a functional MRI study. J Neurophysiol 76:617-621.

Kermadi I, Joseph JP (1995) Activity in the caudate nucleus of monkey during spatial sequencing. J Neurophysiol 74:911-933.

Luppino G, Matelli M, Camarda R, Rizzolatti G (1993) Corticocortical connections of area F3 (SMA-proper) and area F6 (pre-SMA) in the macaque monkey. J Comp Neurol 338:114-140.

Maguire EA, Frackowiak RSJ, Frith CD (1997) Recalling routes around London: activation of the right hippocampus in taxi drivers. J Neurosci 15:7103-7110.

Miyachi S, Hikosaka O, Miyashita K, Kárádi Z, Rand MK (1997) Differential roles of monkey striatum in learning of sequential hand movement. Exp Brain Res 115:1-5.

Mushiake H, Strick PL (1995) Pallidal neuron activity during sequential arm movements. J Neurophysiol 74:2754-2758.

Nakamura K, Sakai K, Hikosaka O (1998) Neuronal activity in medial frontal cortex during learning of sequential procedures. J Neurophysiol 80:2671-2687.

Picard N, Strick PL (1996) Motor areas of the medial wall: a review of their location and functional activation. Cereb Cortex 6:342-353.

Rauch SL, Whalen PJ, Savage CR, Curran T, Kendrick A, Brown HD, Bush G, Breiter HC, Rosen BR (1997) Striatal recruitment during an implicit sequence learning task as measured by functional magnetic resonance imaging. Hum Brain Mapp 5:124-132.

Rizzolatti G, Luppino G, Matelli M (1996) The classic supplementary motor area is formed by two independent areas. Adv Neurol 70:45-56.

Sakai K, Hikosaka O, Miyauchi S, Takino R, Sasaki Y, Pütz B (1998) Transition of brain activation from frontal to parietal areas in visuomotor sequence learning. J Neurosci 18:1827-1840.

Tanji J (1996) New concepts of the supplementary motor area. Curr Opin Neurobiol 6:782-787.

Tanji J, Shima K (1994) Role for supplementary motor area cells in planning several movements ahead. Nature 371:413-416.

Wise SP, Di Pellegrino G, Boussaoud D (1992) Primate premotor cor- 
tex: dissociation of visuomotor from sensory signals. J Neurophysiol 68:969-972.

Woods RPW, Cherry SR, Mazziotta JC (1992) Rapid automated algo- rithm for aligning and reslicing PET images. J Comput Assist Tomogr 16:620-633. 